\title{
Exploring the double-slit interference with linearly polarized photons
}

\author{
Wangmei Zha $\odot,{ }^{1}$ James Daniel Brandenburg $\odot,{ }^{2,3, *}$ Lijuan Ruan, ${ }^{3}$ and Zebo Tang $\oplus^{1, \dagger}$ \\ ${ }^{1}$ University of Science and Technology of China, 230026 Hefei, China \\ ${ }^{2}$ Shandong University, 250100 Jinan, China \\ ${ }^{3}$ Brookhaven National Laboratory, New York 11973, USA
}

(Received 2 July 2020; revised 5 November 2020; accepted 19 January 2021; published 26 February 2021)

\begin{abstract}
The linearly polarized quasireal photons from the highly Lorentz-contracted Coulomb fields of relativistic heavy ions can fluctuate to quark-antiquark pairs, scatter off a target nucleus, and emerge as vector mesons. In the process, the two colliding nuclei can serve as slits, forming a Young's double-slit experiment. In addition to the well-known double-slit interference pattern in position space, a similar interference pattern may be expected in polarization space due to the linear polarization of the colliding photons. In this paper, we investigate the interference effect in polarization space as revealed by the asymmetries of the decay angular distribution for vector meson photoprodution in heavy-ion collisions. We find a periodic oscillation pattern with transverse momentum, which can reasonably explain the second-order modulation in azimuth for the $\rho^{0}$ decay observed by the STAR Collaboration.
\end{abstract}

DOI: 10.1103/PhysRevD.103.033007

The double-slit interference experiment plays an essential role in expressing the central puzzle of quantum mechanics-wave-particle complementarity. Such waveparticle duality continues to be challenged [1-5] and explored in a broad range of entities [6-11]. The double-slit experiment could also be performed in relativistic heavy-ion collisions utilizing the vector meson from coherent photoproduction. The highly Lorentz-contracted electromagnetic field accompanied by the heavy nuclei can be viewed as a spectrum of quasireal photons [12]. The photon emitted from one nucleus can fluctuate to a quarkantiquark pair which then scatters off the other nucleus, emerging as a real vector meson. In the production process, the elastic scattering occurs via Pomeron exchange, which imposes a restriction on the production site within one of the two colliding nuclei. This indicates that the production source consists of two well-separated nuclei (two slits). There are two possibilities: Either nucleus 1 emits a photon and nucleus 2 acts as a target, or vice versa. The two possibilities are indistinguishable, which present the production process as a perfectly double-slit experiment. Furthermore, for the short-lived vector meson (e.g., $\rho^{0}$ ), it decays before the wave functions from the two slits can overlap, and the interference entity is the complete set of

\footnotetext{
*dbrandenburg.ufl@gmail.com

zbtang@ustc.edu.cn
}

Published by the American Physical Society under the terms of the Creative Commons Attribution 4.0 International license. Further distribution of this work must maintain attribution to the author(s) and the published article's title, journal citation, and DOI. Funded by SCOAP ${ }^{3}$. decay products in an entangled state, which is an illustration of the Einstein-Podolsky-Rosen paradox. The double-slit interference effect of photonuclear vector meson production in momentum space has been proposed by Klein and Nystrand [13] and verified by the STAR Collaboration through measurements of the $\rho^{0}$ in ultraperipheral collisions (UPCs) [14]. In Refs. [15,16], we extended the scenario to hadronic heavy-ion collisions and proposed to exploit the strong interactions in the overlap region to test the observation effect on the interference.

The photons generated from the highly Lorentzcontracted electromagnetic field are expected to be fully linearly polarized. It has been suggested by $\mathrm{Li}$ et al. [17] that the collisions of the linearly polarized photons $\left(\gamma+\gamma \rightarrow l^{+}+l^{-}\right)$result in the second-order and fourthorder modulations in azimuth (in the plane perpendicular to the beam direction) between the pair momentum and the lepton momentum for dilepton production. The experimental signature was confirmed by the STAR Collaboration for the dielectron measurements [18]. The vector meson production from linearly polarized photons also possesses a distinctive signature in the asymmetries of the decay angular distributions. Continuous efforts [19-26] has been made for more than 50 years to utilize the linearly polarized photons in vector meson photoproduction as a parity filter for the exchange of particles in the $t$ channel, which is an effective tool to separate the natural from unnatural parity exchange in the $t$ channel. Since the photons are in a linear polarization state, the interference can reveal itself in polarization space, inducing an asymmetry in the decay angular distributions. In this paper, we exploit the linearly polarized photons to investigate the interference pattern in 


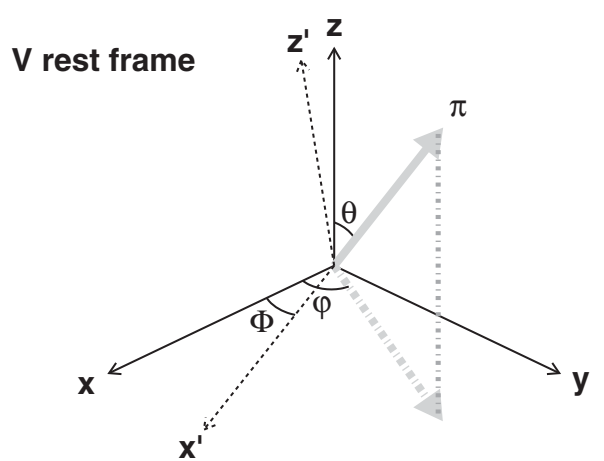

FIG. 1. The coordinate system for the measurement of a twobody decay angular distribution in the vector meson rest frame. The definition of the $x, y$, and $z$ axes is described in the text. The $z^{\prime}$ axis denotes the beam direction, and the $x^{\prime}$ axis represents the linear polarization vector.

polarization space for the vector meson photoproduction in heavy-ion collisions and demonstrate that the signature is a periodic oscillation in asymmetries of the decay angular distributions with transverse momentum.

Hereinafter, we take the process of $\gamma+A \rightarrow V+A \rightarrow$ $d^{+}+d^{-}+A$ in heavy-ion collisions to illustrate the interference effect on the asymmetries of the decay. The decay distribution depends on the choice of the coordinate system, with respect to which the momentum of one of the two decay products is expressed in spherical coordinates. Herein, the right-handed coordinate system for vector meson decay is built up as follows: The $z$ axis is chosen to the direction of flight of the vector meson in the photonnucleon center of mass frame; the $y$ axis is normal to the photoproduction plane; and the $x$ axis is given by $y \times z$. As illustrated in Fig. 1, the decay angles $\theta$ and $\phi$ are the polar and azimuthal angles, respectively, of the unit vector $\tilde{\pi}$, which denotes the direction of flight of one of the decay particles in the vector meson rest frame. In experiment, the direction of the $z$ axis is approximated by that of incoming beams (shown as the $z^{\prime}$ axis in Fig. 1). It has been verified by Monte Carlo calculations that this is a good approximation. Here, we adopt the approximation in our calculation for direct comparisons with experimental results. The angle $\Phi$ shown in Fig. 1 denotes the angle between the photon polarization plane and vector meson production plane.

Under the helicity no-flip assumption, the vector meson inherits the photon polarization state, which is fully linearly polarized. The helicity conservation assumption has been investigated by various experimental measurements [20-25]. Following Ref. [27] and the derivation in the Appendix, the decay angular distribution of vector meson to two spinless products (e.g., $\rho^{0} \rightarrow \pi^{+}+\pi^{-}$) is

$$
\frac{d^{2} N}{d \cos \theta d \phi}=\frac{3}{8 \pi} \sin ^{2} \theta[1+\cos 2(\phi-\Phi)]
$$

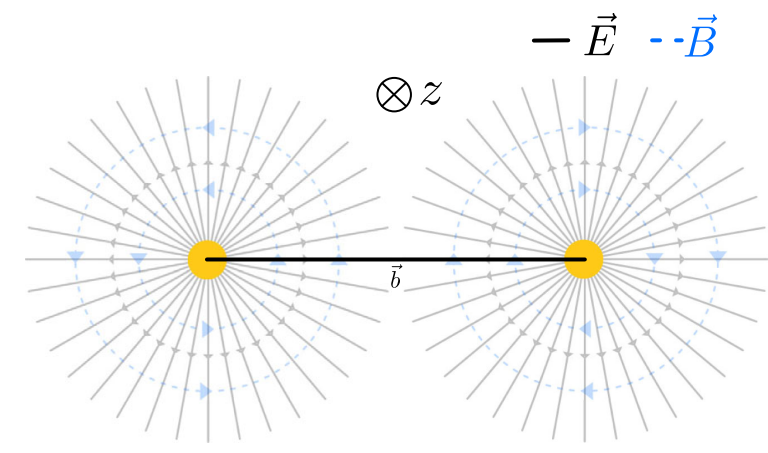

FIG. 2. Schematic diagram for the direction of electric vector of the photons, which hit on the target nuclei, in ultraperipheral heavy-ion collisions.

and to spin $1 / 2$ products (e.g., $\rho^{0} \rightarrow e^{+}+e^{-}$) gives

$\frac{d^{2} N}{d \cos \theta d \phi}=\frac{3}{16 \pi}\left(1+\cos ^{2} \theta\right)\left[1-\frac{\sin ^{2} \theta}{1+\cos ^{2} \theta} \cos 2(\phi-\Phi)\right]$.

As revealed in Eqs. (1) and (2), the linearly polarized states result in the second-order modulations in azimuth, and the strength of the second-order modulation is given by

$$
2\langle\cos (2 \phi)\rangle=\cos (2 \Phi)
$$

and

$$
2\langle\cos (2 \phi)\rangle=-\frac{\sin ^{2} \theta}{1+\cos ^{2} \theta} \cos (2 \Phi),
$$

for the $\rho^{0} \rightarrow \pi^{+}+\pi^{-}$and $\rho^{0} \rightarrow e^{+}+e^{-}$cases, respectively. The modulation strength is determined by the direction of the linear polarization, and the orientation of the photon polarization is determined by the direction of the electric field vector. As demonstrated in Fig. 2, the electric field vector is parallel to the impact parameter at leading order. That is to say that the modulations of the decay distribution for vector meson are determined by the anisotropy in its two-dimensional transverse momentum distribution with respect to the impact parameter at leading order. The variation of polarization direction due to the finite size of nuclei should be small in ultraperipheral collisions, which could be investigated in future work.

The two-dimensional transverse momentum distribution of the vector meson from coherent photoproduction can be obtained by performing a Fourier transformation of the coordinate space amplitude:

$\frac{d^{2} P}{d p_{x} d p_{y}}=\left|\frac{1}{2 \pi} \int d^{2} x_{\perp}\left(A_{1}\left(x_{\perp}\right)+A_{2}\left(x_{\perp}\right)\right) e^{i p_{\perp} \cdot x_{\perp}}\right|^{2}$,

where $A_{1}\left(x_{\perp}\right)$ and $A_{2}\left(x_{\perp}\right)$ are the amplitude distributions in the transverse plane for the two colliding nuclei. Consider, 
(a)

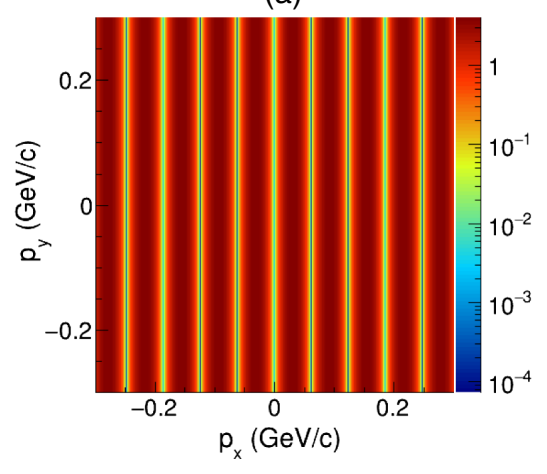

(b)

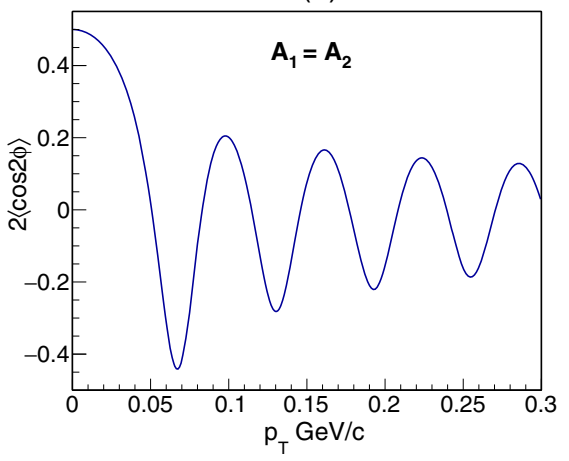

(c)

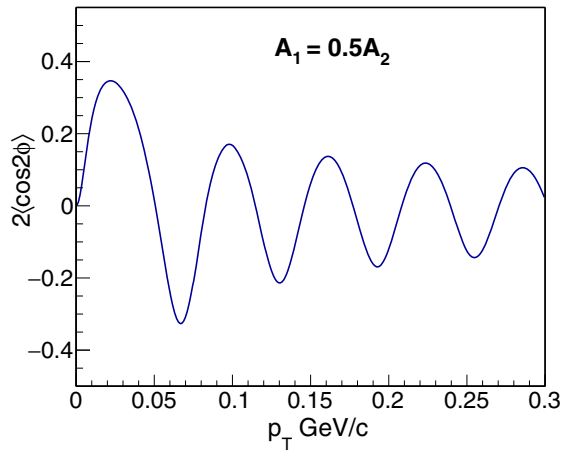

FIG. 3. (a) Two-dimensional transverse momentum distribution pattern with pointlike assumption at midrapidity. (b) The modulation strength $2\langle\cos \phi\rangle$ as a function of transverse momentum at midrapidity. (c) $2\langle\cos \phi\rangle$ as a function of transverse momentum at forward rapidity $\left(A_{1} \neq A_{2}\right)$.

to leading order, we can approximate the production over the two nuclei as two point sources: $A_{1,2}\left(x_{\perp}\right)=$ $\pm A_{1,2} \delta\left(x_{\perp} \pm \frac{1}{2} b\right)$, where $A_{1}$ and $A_{2}$ are the relative amplitudes for the two colliding nuclei, $\delta$ is the Dirac $\delta$ function, $b$ is the impact parameter, and the minus sign in $A_{2}\left(x_{\perp}\right)$ results from the negative parity of the vector meson. With the interference effect, as demonstrated in Fig. 3(a) for the case with $b=20 \mathrm{fm}$ at midrapidity $\left(A_{1}=A_{2}\right)$, the transverse momentum reveals a typical Young's double-slit interference pattern with a series of alternating light and dark fringes, which corresponds to significant asymmetries in azimuth. According to Eq. (3), the modulation strength factor of vector meson decay to two spinless products, $2\langle\cos (2 \phi)\rangle$, versus transverse momentum $\left(p_{T}\right)$ can be extracted from the two-dimensional distribution. The result is shown in Fig. 3(b). The modulation strength shows a periodic oscillation like the hyperbolic Bessel functions with the transverse momentum. The oscillation cycle is determined by the distance between the two source (impact parameter). For production at forward rapidity in heavy-ion collisions, the relative amplitudes from the two nuclei are not equal. Figure 3(c) shows the modulation strength as a function of transverse momentum with unequal amplitudes from the two sources. Since the interference is not complete in this case, the oscillation level is weaker than that at midrapidity. Furthermore, the modulation strength is 0 at $p_{T}=0$, while it is maximum for the case at midrapidity. This results since the interference contribution is close to 0 for $p_{T}=0$.

As mentioned herein before, we have qualitatively demonstrated the interference effect on the asymmetries of the decay angular distributions for vector meson photoproduction. The quantitative calculations are described hereinafter. Following Ref. [15], the production amplitude distribution for vector meson photoproduction is determined by the spatial photon flux and the corresponding $\gamma A$ scattering amplitude $\Gamma_{\gamma A \rightarrow V A}$. According to equivalent photon approximation, the spatial photon flux generated by the heavy nuclei can be written as

$$
\begin{aligned}
n\left(\omega, \vec{x}_{\perp}\right) & =\frac{4 Z^{2} \alpha}{\omega_{\gamma}}\left|\int \frac{d^{2} \vec{k}_{\gamma \perp}}{(2 \pi)^{2}} \vec{k}_{\gamma \perp} \frac{F_{\gamma}\left(\vec{k}_{\gamma}\right)}{\left|\vec{k}_{\gamma}\right|^{2}} e^{i \vec{x}_{\perp} \cdot \vec{k}_{\gamma \perp}}\right|^{2}, \\
\vec{k}_{\gamma} & =\left(\vec{k}_{\gamma \perp}, \frac{\omega_{\gamma}}{\gamma_{c}}\right), \quad \omega_{\gamma}=\frac{1}{2} M_{V} e^{ \pm y},
\end{aligned}
$$

where $\vec{x}_{\perp}$ and $\vec{k}_{\gamma \perp}$ are two-dimensional photon position and momentum vectors, respectively, perpendicular to the beam direction, $\omega_{\gamma}$ is the energy of the emitted photon, $Z$ is the electric charge of the nucleus, $\alpha$ is the electromagnetic coupling constant, $\gamma_{c}$ is the Lorentz factor of the beam, $M_{V}$ and $y$ are the mass and rapidity, respectively, of the vector meson, and $F_{\gamma}\left(\vec{k}_{\gamma}\right)$ is the nuclear electromagnetic form factor. The form factor can be obtained via the Fourier transformation of the charge density in the nucleus. The charge density profile of the nucleus can be parametrized by the Woods-Saxon distribution:

$$
\rho_{A}(r)=\frac{a^{0}}{1+\exp \left[\left(r-R_{\mathrm{WS}}\right) / d\right]},
$$

where the radius $R_{\mathrm{WS}}$ and skin depth $d$ are based on fits to electron-scattering data [28] and $a^{0}$ is the normalization factor. As demonstrated in Eq. (6), the polarization direction for the quasireal photons follows the position vector $\vec{x}_{\perp}$.

The scattering amplitude $\Gamma_{\gamma A \rightarrow V A}$ with the shadowing effect can be estimated via the Glauber [29] + vector meson dominance (VMD) approach [30]:

$$
\Gamma_{\gamma A \rightarrow V A}\left(\vec{x}_{\perp}\right)=\frac{f_{\gamma N \rightarrow V N}(0)}{\sigma_{V N}} 2\left[1-\exp \left(-\frac{\sigma_{V N}}{2} T^{\prime}\left(\vec{x}_{\perp}\right)\right)\right],
$$


where $f_{\gamma N \rightarrow V N}(0)$ is the forward-scattering amplitude for $\gamma+N \rightarrow V+N$ and $\sigma_{V N}$ is the total $V N$ cross section. To account for the coherent length effect, the modified thickness function $T^{\prime}\left(\vec{x}_{\perp}\right)$ is defined as

$T^{\prime}\left(\vec{x}_{\perp}\right)=\int_{-\infty}^{+\infty} \rho\left(\sqrt{\vec{x}_{\perp}^{2}+z^{2}}\right) e^{i q_{L} z} d z, \quad q_{L}=\frac{M_{V} e^{y}}{2 \gamma_{c}}$,

where $q_{L}$ is the longitudinal momentum transfer required to produce a real vector meson. The $f_{\gamma N \rightarrow V N}(0)$ can be determined from the measurements of forward-scattering cross section $\left.\frac{d \sigma_{Y N \rightarrow V N}}{d t}\right|_{t=0}$, which is well parametrized in Ref. [31]. Using the optical theorem and VMD relation, the total cross for $V N$ scattering can be given by

$$
\sigma_{V N}=\frac{f_{V}}{4 \sqrt{\alpha} C} f_{\gamma V \rightarrow V N}
$$

where $f_{V}$ is the $V$-photon coupling and $C$ is a correction factor for the nondiagonal coupling through higher mass vector mesons. Finally, the production amplitude can be given by

$$
A\left(\overrightarrow{x_{\perp}}\right)=\Gamma_{\gamma A \rightarrow V A} \sqrt{n\left(\omega, \vec{x}_{\perp}\right)} .
$$

In addition to the coherent production with interference, there are also an incoherent contribution in heavy-ion collisions, which is significant at relative high transverse momentum. The incoherent production should have no contribution to the asymmetries of decay distribution, which would weaken the overall modulation strength. Similarly to the case of coherent vector meson photoproduction on nuclei, the incoherent cross section $\sigma_{\gamma A \rightarrow V A^{\prime}}$ could be related to the exclusive $\gamma p$ cross section $\sigma_{\gamma p \rightarrow V p}$ via the Glauber + VMD approach. Here, $A^{\prime}$ is the final nuclear state containing products of the nuclear disintegration. The approach gives

$$
\sigma_{\gamma A \rightarrow V A^{\prime}}=\sigma_{\gamma p \rightarrow V p} \int d^{2} \vec{x}_{\perp} T\left(\vec{x}_{\perp}\right) e^{-(1 / 2) \sigma_{V N}^{\mathrm{in}} T\left(\vec{x}_{\perp}\right)},
$$

where $\sigma_{V N}^{\text {in }}=\sigma_{V N}-\sigma_{V N}^{2} /\left(16 \pi B_{V}\right)$ is the inelastic vector meson-nucleon cross section and $B_{V}$ is the slope of the $t$ dependence of the $\gamma p \rightarrow V p$ scattering [31].

Here, we focus on the ultraperipheral collisions, where there is no nuclear overlap to reject hadronic background. According to the optical Glauber model [32], the mean number of projectile nucleons that interact at least once in $A+A$ collisions with impact parameter $b$ is

$$
P_{H}(b)=\int d^{2} \vec{x}_{\perp} T\left(\vec{x}_{\perp}-\vec{b}\right)\left\{1-\exp \left[-\sigma_{N N} T\left(\vec{x}_{\perp}\right)\right]\right\},
$$

where $\sigma_{N N}$ is the total nucleon-nucleon cross section. Then, the probability of having no hadronic interaction is $\exp \left[-P_{H}(b)\right]$. In experiment, the UPC events are usually triggered or selected by the mutual Coulomb dissociation of the nuclei, in which the number of emitted forward neutrons can be detected by zero degree calorimeters. Following the EPA approach, the Coulomb excitation probability of an ultrarelativistic nucleus can be determined by the photon flux accompanied with the nucleus and the appropriate photon-absorption cross section of nuclei. The lowest-order probability for an excitation of nucleus which emits at least one neutron $\left(X_{n}\right)$ is

$$
m_{X n}(b)=\int d \omega n(\omega, b) \sigma_{\gamma A \rightarrow A^{*}}(\omega),
$$

where $n(\omega, b)$ is photon flux described by Eq. (6) and $\sigma_{\gamma A \rightarrow A^{*}}(\omega)$ is the photoexcitation cross section with incident energy $\omega$. The photoexcitation cross section $\sigma_{\gamma A \rightarrow A^{*}}(\omega)$ can be extracted from the experimental measurements [33-39]. The probability of mutual dissociation for the two nuclei with at least one neutron emission for each beam $(X n X n)$ is then given by

$$
P_{X n X n}(b)=\left(1-\exp \left[-m_{X n}(b)\right]\right)^{2} .
$$

As described in our recent work [40], the mutual dissociation probability with any number of neutron emission can be estimated in a similar way.

With the inputs described before, the cross section and differential distributions for vector meson photoproduction can be calculated and can reasonably describe the measurements from STAR [24,41] and ALICE $[42,43]$. The estimated modulation strength $2\langle\cos (2 \phi)\rangle$ of $\rho^{0} \rightarrow \pi^{+}+$ $\pi^{-}$and $\rho^{0} \rightarrow e^{+}+e^{-}$as a function of $p_{T}$ in ultraperipheral $\mathrm{Au}+\mathrm{Au}$ collisions at $\sqrt{s_{N N}}=200 \mathrm{GeV}$ for mutual dissociation mode $X n X n$ are shown in Fig. 4. The modulation strength shows a periodic oscillation with the transverse momentum. The amplitude of the second oscillation period is weaker than that of the first oscillation period, which is due to a larger fraction of incoherent production at higher $p_{T}$. The oscillation feature completely disappears for $p_{T}>0.16 \mathrm{GeV} / \mathrm{c}$, where the incoherent production plays the dominate role in the photoproduction. As expected in Eqs. (3) and (4), the modulation strength for $\rho^{0} \rightarrow e^{+}+e^{-}$ is weaker and possesses opposite sign in comparison with that for $\rho^{0} \rightarrow \pi^{+}+\pi^{-}$. Furthermore, it depends on the acceptance, and the calculations for $\rho^{0} \rightarrow e^{+}+e^{-}$are performed with STAR acceptance as listed in the figure. The results for $\rho^{0} \rightarrow \pi^{+}+\pi^{-}$can reasonably describe the transverse momentum dependence of the secondorder modulation in azimuth observed by the STAR Collaboration [44]. The predictions for $\rho^{0} \rightarrow e^{+}+e^{-}$call for further experimental testing. 


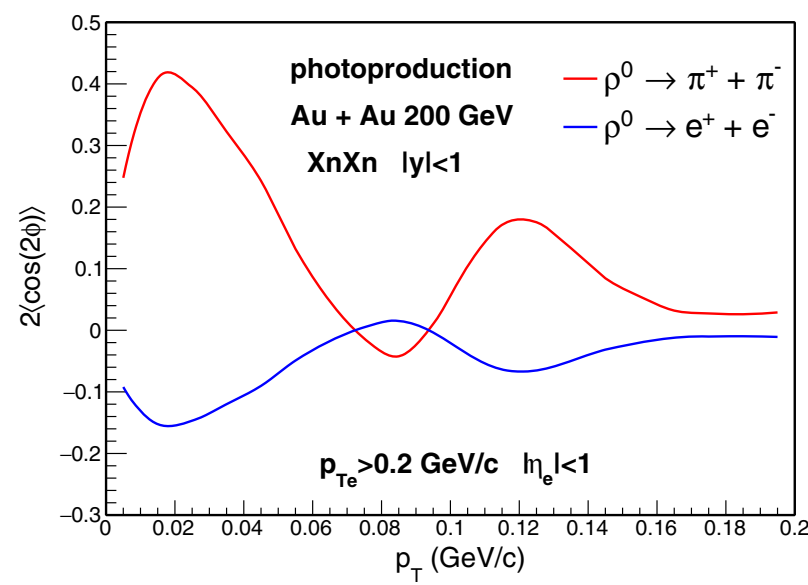

FIG. 4. The modulation strength $2\langle\cos (2 \phi)\rangle$ of $\rho^{0} \rightarrow \pi^{+}+\pi^{-}$ and $\rho^{0} \rightarrow e^{+}+e^{-}$as a function of transverse momentum in ultraperipheral $\mathrm{Au}+\mathrm{Au}$ collisions at $\sqrt{s_{N N}}=200 \mathrm{GeV}$ for mutual dissociation mode $X n X n$.

In summary, the double-slit interference pattern is explored in polarization space with the linearly polarized photons in heavy-ion collisions. We demonstrate how the interference between the two colliding nuclei affects the asymmetries of the decay angular distributions for vector meson photoproduction from linearly polarized photons. Using the vector meson dominance with the Glauber approach, the second-order modulation in azimuth for vector meson decay from photoproduction in ultraperipheral $\mathrm{Au}+\mathrm{Au}$ collision at $\sqrt{s_{N N}}=200 \mathrm{GeV}$ is estimated and reveals a periodic oscillation with transverse momentum. The results for $\rho^{0} \rightarrow \pi^{+}+\pi^{-}$can reasonably describe the decay asymmetries observed by the STAR Collaboration, while the predictions for $\rho^{0} \rightarrow e^{+}+e^{-}$call for further experimental verification. Furthermore, in principle, the modulation strength should also reveal itself for photoproduction in multislit interference setups (future electron-ion collider experiments), which may serve as a novel tool to probe the gluon distribution in nuclei.

\section{ACKNOWLEDGMENTS}

This work was funded by the National Natural Science Foundation of China under Grants No. 11775213 and No. 11720101001, and MOST under Grant No. 2018YFE0104900.

\section{APPENDIX: GENERAL DECAY ANGULAR DISTRIBUTION OF VECTOR MESON TWO-BODY DECAY}

For single production, the vector meson is formed as a superposition of the three $J=1$ eigenstates, $J_{z}=+1,-1,0$ with respect to the polarization axis $z$ :

$$
|V\rangle=a_{+1}|+1\rangle+a_{-1}|-1\rangle+a_{0}|0\rangle .
$$

The calculation is performed in the vector meson rest frame, where the common direction of the two decay products defines the reference axis $z^{\prime}$, oriented conventionally along the direction of the positive product. For the decay of a vector meson to two spinless products (e.g., $\rho^{0} \rightarrow \pi^{+}+\pi^{-}$), the decay system has angular momentum projection 0 along $z^{\prime}$; while for the decay to a dilepton system (e.g., $\rho^{0} \rightarrow e^{+}+e^{-}$), due to helicity conservation for fermions in $\mathrm{QED}$, it has angular momentum projection \pm 1 along $z^{\prime}$. The decay system can be represented as an eigenstate of $J_{z^{\prime}},\left|d^{+} d^{-} ; 1, l^{\prime}\right\rangle$ with $l^{\prime}=+1,-1$, or 0 . The eigenstate along $z^{\prime}$ can be expressed by a superposition of eigenstates of $J_{z},\left|d^{+} d^{-} ; 1, l\right\rangle$ with $l=0, \pm 1$ through the rotation transformation:

$$
\left|d^{+} d^{-} ; 1, l^{\prime}\right\rangle=\sum_{l=0, \pm 1} D_{l l^{\prime}}^{1}(\phi, \theta,-\phi)\left|d^{+} d^{-} ; 1, l\right\rangle
$$

The complex rotation matrix elements $D_{l l^{\prime}}^{1}$ are defined as

$$
D_{l l^{\prime}}^{1}(\phi, \theta,-\phi)=e^{-i\left(l-l^{\prime}\right) \phi} d_{l l^{\prime}}^{1}(\theta) .
$$

The amplitude of the partial process $V(|1, m\rangle) \rightarrow d^{+}+$ $d^{-}\left(\left|1, l^{\prime}\right\rangle\right)$ can then be written as

$$
\begin{aligned}
B_{m l^{\prime}} & =\sum_{l=0, \pm 1} D_{l l^{\prime}}^{1 *}(\phi, \theta,-\phi)\left\langle d^{+} d^{-} ; 1, l|M| V ; 1, m\right\rangle \\
& =B D_{m l^{\prime}}^{1 *}(\phi, \theta,-\phi) .
\end{aligned}
$$

Here we imposed $\left\langle d^{+} d^{-} ; 1, l|M| V ; 1, m\right\rangle=B \delta_{m l}$ according to the angular momentum conservation and rotational invariance ( $B$ is independent of $m$ ). The total amplitude of $V \rightarrow d^{+}+d^{-}\left(\left|1, l^{\prime}\right\rangle\right)$ with the superposition of eigenstate written by Eq. (A1) is

$$
B_{l^{\prime}}=\sum_{m=0, \pm 1} a_{m} B D_{m l^{\prime}}^{1 *}(\phi, \theta,-\phi) .
$$

The probability of the transition is obtained by squaring Eq. (A5) and summing over the spin alignments of the decay system. For photoproduction, the vector meson inherits the photon polarization state. which is fully linearly polarized. It reads

$$
|V\rangle=-\frac{1}{\sqrt{2}} e^{-i \Phi}|+1\rangle+\frac{1}{\sqrt{2}} e^{i \Phi}|-1\rangle,
$$

where $\Phi$ is the angle between the linear polarization vector and the production plane of vector meson. This gives $a_{0}=0, a_{+1}=-\frac{1}{\sqrt{2}} e^{-i \Phi}$, and $a_{-1}=\frac{1}{\sqrt{2}} e^{i \Phi}$. For the decay 
of a vector meson to two spinless products (e.g., $\rho^{0} \rightarrow \pi^{+}+\pi^{-}$), with $d_{00}^{1}=\cos \theta$ and $d_{ \pm 10}^{1}=\mp \frac{1}{\sqrt{2}} \sin \theta$, the decay distribution can be written as

$W(\cos \theta, \phi) \propto\left|B_{0}\right|^{2} \propto \frac{3}{8 \pi} \sin ^{2} \theta[1+\cos 2(\phi-\Phi)]$.

For the decay to a dilepton system (e.g., $\rho^{0} \rightarrow e^{+}+e^{-}$), with $d_{0 \pm 1}^{1}= \pm \frac{1}{\sqrt{2}} \sin \theta, d_{ \pm 1 \pm 1}^{1}=\frac{1+\cos \theta}{2}$, and $d_{\mp 1 \pm 1}^{1}=\frac{1-\cos \theta}{2}$, the decay distribution can be written as
$W(\cos \theta, \phi)$

$\propto \sum_{l^{\prime}= \pm 1}\left|B_{l^{\prime}}\right|^{2}$

$\propto \frac{3}{16 \pi}\left[1+\cos ^{2} \theta-\sin ^{2} \theta \cos 2(\phi-\Phi)\right]$

$\propto \frac{3}{16 \pi}\left(1+\cos ^{2} \theta\right)\left[1-\frac{\sin ^{2} \theta}{1+\cos ^{2} \theta} \cos 2(\phi-\Phi)\right]$.
[1] M. Scully et al., B.-G. Englert, and H. Walther, Nature (London) 351, 111 (1991).

[2] H. Wiseman and F. Harrison, Nature (London) 377, 584 (1995).

[3] F. Lindner, Phys. Rev. Lett. 95, 040401 (2005).

[4] M. Kiffner, J. Evers, and C. H. Keitel, Phys. Rev. Lett. 96, 100403 (2006).

[5] B. King, A. Di Piazza, and C. H. Keitel, Nat. Photonics 4, 92 (2010).

[6] C. Jönsson, Z. Phys. 161, 454 (1961).

[7] A. Zeilinger, R. Gähler, C. G. Shull, W. Treimer, and W. Mampe, Rev. Mod. Phys. 60, 1067 (1988).

[8] O. Carnal and J. Mlynek, Phys. Rev. Lett. 66, 2689 (1991).

[9] M. Arndt, O. Nairz, J. Vos-Andreae, C. Keller, G. van der Zouw, and A. Zeilinger, Nature (London) 401, 680 (1999).

[10] M. R. Andrews et al., Science 275, 637 (1997).

[11] L. Hackermüller, S. Uttenthaler, K. Hornberger, E. Reiger, B. Brezger, A. Zeilinger, and M. Arndt, Phys. Rev. Lett. 91, 090408 (2003).

[12] F. Krauss, M. Greiner, and G. Soff, Prog. Part. Nucl. Phys. 39, 503 (1997).

[13] S. R. Klein and J. Nystrand, Phys. Rev. Lett. 84, 2330 (2000).

[14] B. I. Abelev et al. (STAR Collaboration), Phys. Rev. Lett. 102, 112301 (2009).

[15] W. Zha, S. R. Klein, R. Ma, L. Ruan, T. Todoroki, Z. Tang, Z. Xu, C. Yang, Q. Yang, and S. Yang, Phys. Rev. C 97, 044910 (2018).

[16] W. Zha, L. Ruan, Z. Tang, Z. Xu, and S. Yang, Phys. Rev. C 99, 061901 (2019).

[17] C. Li, J. Zhou, and Y.-j. Zhou, Phys. Lett. B 795, 576 (2019).

[18] J. Adam et al. (STAR Collaboration), arXiv:1910.12400.

[19] K. Schilling and F. Storim, Nucl. Phys. B7, 559 (1968).

[20] L. Criegee et al., Phys. Lett. B 28, 282 (1968).

[21] J. Ballam et al., Phys. Rev. Lett. 24, 1364 (1970).

[22] Y. Eisenberg, B. Haber, E. Kogan, U. Karshon, E. E. Ronat, A. Shapira, and G. Yekutieli, Nucl. Phys. B104, 61 (1976).

[23] M. Derrick et al., Phys. Lett. B 377, 259 (1996).
[24] B. I. Abelev et al. (STAR Collaboration), Phys. Rev. C 77, 034910 (2008).

[25] T. Hiraiwa et al. (LEPS Collaboration), Phys. Rev. C 97, 035208 (2018).

[26] H. Xing et al., J. High Energy Phys. 10 (2020) 064.

[27] K. Schilling, P. Seyboth, and G. Wolf, Nucl. Phys. B15, 397 (1970).

[28] R. C. Barrett and D. F. Jackson, Nuclear Sizes and Structure (Oxford University Press, Oxford, 1977).

[29] M. L. Miller, K. Reygers, S. J. Sanders, and P. Steinberg, Annu. Rev. Nucl. Part. Sci. 57, 205 (2007).

[30] T. H. Bauer, R. D. Spital, D. R. Yennie, and F. M. Pipkin, Rev. Mod. Phys. 50, 261 (1978).

[31] S. R. Klein, J. Nystrand, J. Seger, Y. Gorbunov, and J. Butterworth, Comput. Phys. Commun. 212, 258 (2017).

[32] M. L. Miller, K. Reygers, S. J. Sanders, and P. Steinberg, Annu. Rev. Nucl. Part. Sci. 57, 205 (2007).

[33] A. Veyssiere, H. Beil, R. Bergere, P. Carlos, and A. Lepretre, Nucl. Phys. A159, 561 (1970).

[34] A. Leprêtre, H. Beil, R. Bergère, P. Carlos, J. Fagot, A. De Miniac, and A. Veyssière, Nucl. Phys. A367, 237 (1981).

[35] P. Carlos, H. Beil, R. Bergére, J. Fagot, A. Leprêtre, A. De miniac, and A. Veyssiére, Nucl. Phys. A431, 573 (1984).

[36] T. A. Armstrong et al., Phys. Rev. D 5, 1640 (1972).

[37] D. O. Caldwell, V. B. Elings, W. P. Hesse, R. J. Morrison, F. V. Murphy, and D. E. Yount, Phys. Rev. D 7, 1362 (1973).

[38] S. Michalowski, D. Andrews, J. Eickmeyer, T. Gentile, N. Mistry, R. Talman, and K. Ueno, Phys. Rev. Lett. 39, 737 (1977).

[39] T. Armstrong et al., Nucl. Phys. B41, 445 (1972).

[40] J. D. Brandenburg et al., arXiv:2006.07365.

[41] L. Adamczyk et al. (STAR Collaboration), Phys. Rev. C 96, 054904 (2017).

[42] J. Adam et al. (ALICE Collaboration), J. High Energy Phys. 09 (2015) 095.

[43] S. Acharya et al. (ALICE Collaboration), J. High Energy Phys. 06 (2020) 035.

[44] D. Brandenburg (STAR Collaboration), Quark Matter 2019 (2019), https://indico.cern.ch/event/792436/contributions/ $3535864 /$. 